\title{
次
}

Jorge Infante González*

\section{EL DEBATE SOBRE LA REGULACIÓN DE PLATAFORMAS DIGITALES EN EL SIGLO XXI}

El objetivo del presente artículo es presentar el estado actual del debate y el futuro tratamiento regulatorio de las plataformas digitales. Para ello se analizan sus principales características, su impacto social y económico, y se describen las líneas del debate en diversas áreas geográficas, con especial énfasis en el caso europeo. En el artículo se indican los principales instrumentos regulatorios, consultas públicas e informes que están siendo usados como referencia del debate regulatorio que configurará en el próximo año el modelo de intervención e institucional en Europa.

Palabras clave: ley de servicios digitales, competencia, DSA, gatekeeper, intermediación mercados, NCT, servicios digitales.

Clasificación JEL: K23, L13, L43.

\section{Introducción}

En el mundo del siglo xxI las plataformas digitales se han convertido en un elemento clave de nuestra actividad, tanto en el ámbito económico como en el ámbito social. Nos apoyamos en ellas para buscar información, acceder a contenidos, comprar y vender productos y servicios, informarnos, buscar empleo, contratar los viajes o mantener nuestras relaciones sociales. En cierta medida, actúan durante una parte relevante de nuestra rutina diaria como las ventanas a través de las

*Experto en Dirección de Telecomunicaciones y Audiovisual. Comisión Nacional de los Mercados y la Competencia.

Versión de octubre de 2020.

DOI: https:/doi.org/10.32796/bice.2020.3129.7114

Este artículo refleja exclusivamente la posición sobre el debate regulatorio de su autor. Cualquier error u omisión es responsabilidad exclusiva del mismo. cuales nos relacionamos con otras personas y con las empresas que nos proporcionan servicios y productos.

El uso creciente de las plataformas digitales se ha visto incrementado aún más en los últimos años por el uso extendido de terminales móviles y tabletas, así como el éxito en la extensión de la banda ancha. Para hacerse una idea de su importancia, la capitalización bursátil combinada de las cinco mayores plataformas (Google, Amazon, Facebook, Apple y Microsoft) fue de alrededor de 4 billones de euros, en 2019, que se corresponde con cerca de la cuarta parte del producto interior bruto de la UE (Comisión Europea, 2020c).

Las plataformas digitales han aportado y aportan cada vez más ventajas a usuarios finales y empresas, dado que reducen los costes de transacción y permiten acceder de $D$ 
forma sencilla a productos y servicios difícilmente accesibles en el mundo físico, suministrando servicios innovadores y útiles. No obstante, las características que hacen de las plataformas digitales herramientas muy potentes, para la intermediación de bienes y servicios y facilitar la comunicación entre los propios ciudadanos y estos con las empresas, hacen también que puedan aparecer efectos indeseados en aspectos muy relevantes como el dinamismo de la competencia, la innovación futura, la protección de consumidores frente a contenidos dañinos o publicidad indebida, la privacidad o la libertad de expresión. Por este motivo, las plataformas digitales plantean nuevos retos regulatorios muy relevantes. El presente artículo pretende mostrar los principales retos, así como el estado actual del debate regulatorio, especialmente en Europa.

\section{Qué son las plataformas digitales y sus características económicas}

Las plataformas digitales son servicios que se apoyan en infraestructura informática y de comunicaciones para facilitar la interacción entre dos o más grupos distintos de usuarios que interaccionan entre ellos usando estos servicios digitales. Como se verá más adelante, el aspecto intermediador de las plataformas, junto con el hecho de que dicha intermediación se produzca a través de medios electrónicos, son clave para comprender sus propiedades económicas.

Bajo esta amplia definición están incluidos servicios como la búsqueda en internet (como Google Search o Bing), las redes sociales (como Facebook o Twitter), los mercados virtuales de venta de productos (como Amazon o Alibaba), de venta de servicios especializados (como Booking), de adquisición de apps (como las tiendas de aplicaciones de Google y Apple) y cualquier otra plataforma que actúe de intermediario entre grupos distintos. En OCDE (2019) se puede encontrar una caracterización tipológica de las diversas plataformas, así como un completo anexo que analiza y proporciona datos sobre las plataformas más relevantes a día de hoy.

Las plataformas digitales presentan una combinación de propiedades que llevan tanto a efectos deseables como a efectos que pueden ser dañinos a largo plazo para sus usuarios.

En primer lugar, la intermediación entre varios grupos de usuarios hace que se produzcan externalidades indirectas. Esto es, cuanto más miembros hay en un grupo (por ejemplo, compradores), más valor tiene la plataforma para el otro grupo (por ejemplo, vendedores).

El hecho de que la intermediación que realizan estas plataformas se produzca sobre una infraestructura digital permite reducir enormemente los costes de transacción para todos los actores involucrados y plantear importantes economías de escala y ámbito. La digitalización de las transacciones y el carácter digital y personalizado de muchos de los servicios que se prestan hacen también que sea rápido y de bajo coste recopilar una gran cantidad de datos sobre transacciones, usuarios finales y negocios que usan las plataformas. Estos datos permiten a las plataformas digitales disfrutar de ventajas competitivas importantes respecto a sus competidores.

Por último, muchas de las plataformas digitales aplican estrategias de empaquetado, configurando ecosistemas en donde se ofrecen servicios complementarios a sus usuarios en muy diversos ámbitos. Los ecosistemas de Google (búsquedas, mapas, apps, correo, videoconferencia, etcétera) o Amazon Prime $\triangleright$ 
(venta de productos, streaming de vídeo, música, etc.) son buenos ejemplos en este sentido.

La combinación de estos factores hace que una parte de estas plataformas puedan actuar como socios comerciales insoslayables (gatekeepers en terminología anglosajona) para las transacciones que se llevan a cabo a través de la plataforma. Esta situación plantea un poder significativo de intermediación, que puede derivar en ineficiencias y problemas estructurales que impidan que la sociedad aproveche en todo su potencial las extraordinarias capacidades de las plataformas digitales.

\section{Impacto social y económico de las plataformas digitales}

Las plataformas digitales plantean interesantes retos en diversos aspectos fundamentales como son la libertad de expresión, la protección frente a contenidos perjudiciales, la calidad del debate público, la privacidad, la competencia y la innovación y, en definitiva, la transición hacia una sociedad digital, que están relacionados entre sí, haciendo aún más complejo el diseño de políticas públicas que faciliten la evolución a una sociedad digital que garantice los derechos individuales y de la libertad de expresión, aproveche los potentes medios digitales para no solo mantener, sino también mejorar, la calidad del debate público y, en definitiva, de la democracia, y explote el potencial de los servicios digitales para mejorar el bienestar de los ciudadanos a través de la innovación y la competencia.

El creciente uso de las plataformas digitales como foros públicos de intercambio de ideas plantea multitud de retos, como son asegurar el debate público transparente controlando las informaciones falsas, protegiendo a los ciudadanos y a determinados colectivos, como pueden ser los menores, o evitando el discurso de odio, sin menoscabo de la libertad de expresión, o controlar de manera eficiente el uso ilícito o abusivo de los datos personales de los consumidores.

En lo que respecta a los efectos económicos, hay varios aspectos a tener en cuenta. En primer lugar, el elevado poder de intermediación de las plataformas más grandes supone que pueden favorecer a sus propios productos o servicios cuando participan en la propia plataforma como un actor más (como, por ejemplo, podría ser el caso de los productos de Amazon comercializados en su plataforma), o establecer condiciones abusivas para uno o ambos lados del mercado donde actúan como intermediarios insoslayables. Los denominados gatekeepers, que disponen de este poder elevado de intermediación, disponen de un amplio margen de acción para aprovechar el poder de mercado, ya que no existen plataformas alternativas que se puedan usar por uno u otro lado del mercado, pudiendo actuar en consecuencia de forma independiente de la competencia sin que tampoco los usuarios en ambos lados del mercado en el que intermedian (sean productos físicos, o servicios como las búsquedas o acceso a aplicaciones) puedan disciplinar su comportamiento.

La entrada en el mercado para nuevos actores es otro aspecto a tener en cuenta, ya que las externalidades directas e indirectas, economías de escala y ámbito hacen difícil, en muchos casos, la entrada de nuevos competidores. Los grandes actores, además, participan en procesos de adquisición continua de pequeñas compañías innovadoras que, en determinados casos, pueden suponer limitar la innovación o la aparición de futuros competidores.

Tal como se ha indicado, los factores indicados no son óbice para que las plataformas $\triangle$ 
digitales sean un motor de innovación, creación de nuevos servicios y nuevos modelos de intermediación. La experiencia de crecimiento del uso de plataformas digitales en la pandemia actual es un buen ejemplo de ello. El enorme potencial transformativo social y económico que presentan, junto con su carácter sistémico, obliga a las autoridades de regulación y competencia y a las Administraciones a contemplar la mejor manera de aprovechar todo su potencial y prevenir potenciales problemas para la sociedad en su conjunto.

\section{Debate regulatorio mundial}

Las plataformas digitales llevan tiempo en el punto de mira de las Administraciones y de las autoridades de regulación y competencia, y han sido objeto de casos de competencia muy relevantes en los últimos años, como los casos de Google en la Unión Europea sobre búsquedas de compra (Comisión Europea, 2017), publicidad online (Comisión Europea, 2019a) y el caso Android (Comisión Europea, 2018), o la investigación abierta por posibles prácticas anticompetitivas de Amazon (Comisión Europea, 2019b) o el caso Facebook en Alemania (Bundeskartellamt, 2019). La importancia creciente de las plataformas digitales en la economía, la comunicación interpersonal y el debate público en los últimos años han hecho que Administraciones y autoridades de competencia y regulación presten una especial atención a los retos planteados por estas plataformas, abriéndose un debate sobre nuevas herramientas regulatorias ex ante y ex post para el tratamiento de los problemas estructurales de competencia e impacto social que plantean estas plataformas.

Actualmente, el debate sobre los aspectos regulatorios de las plataformas digitales se está desarrollando en todo el mundo, y con especial intensidad en la Unión Europea, Reino Unido, Estados Unidos y Australia.

Este debate gira sobre varios ejes interrelacionados: regulación de contenidos audiovisuales, regulación ex post de competencia, posible regulación ex ante y regulación de privacidad y acceso y uso de los datos.

En lo que respecta a la regulación de acceso a contenidos y redes sociales, las plataformas digitales están cambiando complemente la forma de acceder a la información de todo tipo e interaccionar entre ciudadanos, así como la forma de acceder a contenidos audiovisuales, pasándose de las emisiones lineales a las plataformas de streaming audiovisual. Los principales temas de debate en este ámbito son el diseño de las medidas más adecuadas para una protección efectiva de los usuarios (muy especialmente los menores) y el régimen de responsabilidad de las plataformas que intermedian contenidos, incluyendo las redes sociales, en lo que respecta a la desinformación, contenidos ilegales, o control del debate público.

El debate sobre aspectos de competencia para plataformas digitales se centra en la adecuación de la regulación actual de competencia para abordar los problemas potenciales que plantean las plataformas digitales, donde muchos actores identifican que los marcos reguladores actuales de antitrust resultan insuficientes para tratar de manera rápida y efectiva problemas estructurales recurrentes. Para ello, se está planteando, entre otros, la extensión de poderes regulatorios para que las autoridades de competencia puedan llevar a cabo investigaciones de mercado sobre plataformas digitales, incluyendo la capacidad de imponer obligaciones (ver más adelante el caso de la CMA) y la implementación de poderes para tratar los problemas estructurales que se presentan $\triangleright$ 
en las plataformas digitales antes de que esté hecho el daño a la competencia, yendo más allá del ámbito de los artículos 101 y 102 del Tratado de Fundación de la Unión Europea. Un tema adicional de debate es la adaptación de la regulación de fusiones y adquisiciones para dar una mayor flexibilidad a las autoridades de competencia, al objeto de evitar las denominadas killer acquisitions por parte de las grandes plataformas que puedan inhibir el desarrollo de la competencia a través de la adquisición de nuevos posibles entrantes. Sobre este aspecto, el reciente informe del centro de estudios CERRE (Bourreau y De Streel, 2020) plantea los principales aspectos involucrados en los análisis de fusiones para plataformas digitales y el debate actual sobre la evolución de su regulación.

Resulta también de interés el debate existente sobre las posibles medidas regulatorias a adoptar, como prohibiciones u obligaciones al objeto de impedir comportamientos indeseados que impidan la competencia efectiva intra e interplataformas. Entre ellas se discute sobre impedir el denominado self-preferencing, donde la plataforma aplica un trato de ventaja a los servicios o productos que comercializa respecto a otros actores que usan la plataforma (por ejemplo, trato preferencial en las búsquedas), o limitaciones al uso de datos de las transacciones realizadas en la plataforma para negocios conexos.

En general, además de revisar la regulación horizontal existente como la directiva de comercio electrónico (Comisión Europea, 2000), se encuentra bajo debate aplicar medidas regulatorias específicas asimétricas para plataformas con gran poder de intermediación o de mercado con un número elevado de usuarios y donde no sea habitual el llamado multi-homing (el usuario no usa una única plataforma, sino que realiza interacciones en varias plataformas, como sería el caso de adquirir productos tanto en Amazon como en Alibaba). Esto es, la acción regulatoria estaría limitada a un número pequeño de grandes plataformas con elevado poder de intermediación.

Tanto la identificación de los «mercados relevantes» de intermediación como el umbral de intervención, esto es, las características que llevan a identificar a una plataforma como gatekeeper, no han sido hasta el momento definidos, más que en sus términos generales (como, por ejemplo, identificación de áreas de negocio como las búsquedas en internet, las tiendas de apps, o umbrales, como un elevado número de usuarios, o tiempo pasado en la plataforma). Este será uno de los aspectos que probablemente se debatirá más en los próximos meses.

El tratamiento regulatorio de ecosistemas compuestos de varias plataformas interrelacionados como Google o Amazon es también objeto de debate (ver, por ejemplo, Condorelli y Padilla, 2020). La regulación de ecosistemas plantea una mayor complejidad que el tratamiento de plataformas aisladas, pudiendo incluirse como posibles medidas la separación estructural o funcional.

Tal como se indicaba, el debate regulatorio sobre plataformas digitales está teniendo lugar en todo el mundo, siendo especialmente vivo e interesante en la Unión Europea, Reino Unido, Estados Unidos y Australia. Los informes más relevantes que están conformando el debate regulatorio son muy recientes, habiendo sido publicados en 2019 y 2020.

En lo que respecta a Reino Unido, el informe «Furman» (Furman et al., 2019), encargado a un panel de expertos por el Gobierno del Reino Unido, fue uno de los primeros en publicarse. En este informe se plantea la creación de una unidad de mercados digitales con poderes para establecer códigos de conducta para $\triangleright$ 
plataformas con elevado poder de intermediación, desarrollo de estándares abiertos, movilidad de datos y acceso a datos no personales. Asimismo, se propone la adaptación de las reglas de control de fusiones para facilitar su escrutinio en el caso de las plataformas digitales y reforzar la aplicación de la regulación de competencia. En el marco de los contenidos digitales, el Gobierno del Reino Unido (2019) ha llevado a cabo un extenso estudio sometido a consulta pública sobre el borrador de planes del Gobierno para reforzar la seguridad de los contenidos online a través de acciones diversas, incluyendo el establecimiento de un marco regulatorio específico para la protección de menores, el tratamiento del discurso de odio y la desinformación.

La Autoridad de la Competencia y los Mercados de Reino Unido (CMA) es citada habitualmente como referencia para una posible implementación de poderes de investigación de mercados en otras autoridades de competencia, como los que dispone la CMA para el tratamiento de problemas de competencia relacionados con plataformas digitales.

El sistema legal británico establece que la CMA, junto con las autoridades de regulación sectoriales, puede llevar investigaciones de mercados enfocadas a identificar si las características del mercado impiden, restringen o distorsionan la competencia. Cuando la CMA identifica que determinadas características del mercado tienen un efecto adverso en la competencia que impacta sobre los consumidores, debe analizar cómo se pueden solucionar e identificar, e implementar las acciones correctoras (remedies) proporcionadas correspondientes. La CMA dispone de amplias competencias para la aplicación de remedies, incluyendo la separación estructural. La identificación de efectos adversos en la competencia no conlleva procedimientos sancionadores, multas ni pago de compensaciones a afectados para las compañías investigadas por su comportamiento en el pasado.

La Comisión Europea encargó un estudio centrado en estos poderes de investigación de mercados de la CMA para analizar su posible aplicación en el ámbito comunitario (Comisión Europea, 2020e). La CMA ha realizado informes interesantes como el estudio de mercado sobre publicidad digital (CMA, 2020), donde recomienda el establecimiento de reglas ex ante para gobernar las actividades de determinadas plataformas activas en la publicidad digital como Google o Facebook. También en Reino Unido la autoridad reguladora de comunicaciones y audiovisual, Ofcom, colabora estrechamente con la CMA en elevar recomendaciones al Gobierno sobre el futuro diseño del marco regulador para plataformas y servicios digitales.

La Comisión Australiana de la Competencia y los consumidores (ACCC, Australian Competition and Consumer Commission) realizó en 2019 una extensa investigación sobre estas plataformas (ACCC, 2019) y el Gobierno australiano se encuentra también en proceso de deliberación sobre la regulación de estas plataformas, trabajando sobre propuestas similares a la británica y europea.

Una parte de la crítica hacia el debate regulatorio en Europa y Australia se basa en atribuir a las correspondientes Administraciones un objetivo no declarado proteccionista, al ser la mayor parte de las grandes plataformas estadounidenses o chinas. Si bien es cierto que Europa carece en general de grandes plataformas $^{1}$, y sin entrar a juzgar objetivos que no $\triangleright$

1 Existiendo, no obstante, excepciones como Booking o Spotify, que, en cualquier caso, no tienen el volumen de los GAFAM (Google, Apple, Facebook, Amazon, Microsoft). 
se explicitan por parte de las autoridades europeas, hay que destacar que las Administraciones de Estados Unidos, donde tienen su sede muchas de las grandes plataformas digitales, también están inmersos en el debate sobre el poder de mercado de las plataformas digitales y la adaptación del marco regulador de la competencia para los retos que plantean. Quizás el informe más influyente y citado realizado en Estados Unidos es el informe Stigler (Morton et al., 2019). Este informe identifica una tendencia hacia la concentración en los mercados digitales, identificando a las plataformas digitales como actores con un elevado poder político. Recomienda revisar los umbrales para el análisis de fusiones, aumentar la transparencia de las plataformas digitales, adoptar reglas pro consumidor, forzar la interoperabilidad en determinados casos, reforzar las reglas de competencia, reducir el poder de los datos, y el establecimiento de una autoridad digital independiente. Para finalizar con el caso de Estados Unidos, el Departamento de Justicia (DoJ) ha abierto, el 20 de octubre, una investigación a Google, acusándola de mantener un monopolio ilegal sobre las búsquedas y los anuncios en estas (DoJ, 2020). El caso se sustenta en la teoría del daño, similar a la del caso Android de la Comisión Europea (CE, 2018).

Por su especial relevancia para España, el caso europeo se trata de forma separada en la siguiente sección.

\section{Debate regulatorio en la Unión Europea}

Las instituciones comunitarias, los Gobiernos de los Estados miembros y los organismos reguladores, tanto de la competencia como de regulación ex ante, o convergentes como es el caso de la CNMC en España, participan también en el debate regulatorio sobre las plataformas digitales, tanto en los aspectos relacionados con los contenidos como en los aspectos económicos. Nos centraremos especialmente en las iniciativas y situación del debate a nivel comunitario, ya que las grandes plataformas presentan una dimensión transnacional, y a lo largo de 2021 es de prever que se fijen reglas que afecten a toda la Unión Europea, con un papel muy relevante en su aplicación para la Comisión Europea. Todo parece indicar que es muy posible que Europa sea, una vez más, referencia mundial en aspectos regulatorios, como sucedió con el Reglamento General de Protección de Datos, ya que la discusión y la agenda regulatoria se encuentran más avanzadas que en otras partes del mundo.

Tal como se ha indicado, las autoridades de competencia europeas, tanto a nivel comunitario (DG-COMP) como a nivel nacional (CNMC en el caso español) han sido muy activas en el tratamiento de casos de competencia relacionados con plataformas digitales. La experiencia adquirida en el tratamiento de estos casos y la importancia creciente de las plataformas digitales han llevado a la apertura de un amplio debate sobre la oportunidad de actualizar la regulación de contenidos para adaptarla a la realidad de las redes sociales, la regulación de competencia para dotar a las autoridades de herramientas adaptadas a las particularidades de las plataformas digitales, así como la posible definición de obligaciones directas o personalizadas a aplicar para las plataformas «sistémicas» que disfruten de un elevado poder de negociación o actúen como socio comercial insoslayable.

Entre los informes elaborados en el ámbito comunitario que están guiando el debate europeo, el más citado es el denominado $\triangleright$ 
informe «Crémer» (Crémer et al., 2019), encargado por la comisaria Vesthager a un panel de expertos. Las conclusiones de este informe, centradas en los aspectos de competencia para las plataformas digitales, han servido de base para el lanzamiento de las consultas públicas sobre el Acta de Servicios Digitales (en adelante, DSA, Digital Services Act) y la denominada New Competition Tool (NCT).

Tanto la consulta pública sobre la DSA (Comisión Europea, 2020b) como la consulta sobre la NCT (Comisión Europea, 2020d) se abrieron en junio de 2020, en febrero en el caso de la DSA (Comisión Europea, 2020b) y en junio en el caso de la NCT, finalizando el plazo de las mismas el 30 de junio para la NCT y el 9 de septiembre para la DSA. Solo la consulta sobre la DSA ha recibido alrededor de tres mil respuestas de todo tipo de actores, públicos y privados.

En el ámbito europeo destacan los detallados informes elaborados en los dos últimos años por el centro de estudios CERRE (Centre on Regulation in Europe) sobre los distintos aspectos contemplados en la DSA, resultando de especial interés los informes sobre implementación de obligaciones complejas, como el acceso a datos (Broughton et al., 2020) y compartición de estos (De Streel y Feasey, 2020). La portabilidad de datos, una posible obligación para facilitar la competencia, se trata en Krämer et al. (2020) y las posibles medidas a aplicar sobre plataformas verticalmente integradas en Feasey y Krämer (2019) y en Bourreau y De Streel (2019).

Las consultas públicas de la Comisión sobre la DSA y la NCT tienen como objetivo el diseño de la propuesta regulatoria de la Comisión Europea, que tendrá que ser sometida a discusión y votación por parte tanto del Parlamento Europeo como del Consejo. La publicación del paquete regulatorio propuesto por la Comisión Europea estaba inicialmente prevista para el primer cuatrimestre de 2021, si bien la Comisión Europea ha indicado que adelantará su publicación para diciembre de 2020. Si se cumple el calendario previsto, es muy posible que a lo largo de 2021 se apruebe el paquete regulatorio europeo.

Este paquete regulatorio tratará los principales aspectos implicados en la regulación de plataformas. En primer lugar, se realizará una propuesta sobre los retos planteados en la regulación de plataformas que intermedian contenidos digitales relativos a desinformación, responsabilidad de las plataformas, gobernanza, principio de país de origen, etc. En este contexto resulta de especial interés la respuesta a la consulta pública de ERGA, el grupo de reguladores europeos de servicios audiovisuales, de la que la CNMC forma parte (ERGA, 2020). En segundo lugar, se tratará la regulación para todas las plataformas digitales de los servicios de comercio electrónico, contemplándose como una de las opciones la actualización y modernización de la Directiva de comercio electrónico vigente desde hace veinte años (Comisión Europea, 2000) ${ }^{2}$.

En tercer lugar, la Comisión Europea se pronunciará sobre el tratamiento regulatorio ex ante de los denominados gatekeepers, optando bien por una lista predefinida de obligaciones y prohibiciones, bien por un conjunto de posibles obligaciones personalizables a cada caso particular, entre las cuales podrían encontrarse obligaciones de compartición de datos, limitaciones a su uso, interoperación o separación funcional o estructural, o una $\triangleright$

\footnotetext{
2 El informe (De Streel y Huvosec, 2020), preparado por los profesores Alexandre de Streel de la Universidad de Namur y Martin Huvosec de la London School of Economics para el comité IMCO, realiza un análisis muy interesante sobre la posible evolución de esta directiva.
} 
combinación de ambas opciones. Sobre la aplicación de un posible marco regulatorio ex ante, es de interés la respuesta del Organismo de Reguladores Europeos de las Comunicaciones Electrónicas (BEREC, 2020), del cual forma parte también la CNMC, que, partiendo de su experiencia en la aplicación del marco regulatorio de comunicaciones electrónicas, ha realizado una propuesta de planteamiento de un marco regulatorio que incluye una combinación de obligaciones y prohibiciones predefinida con un catálogo de obligaciones adaptables a cada caso concreto.

En cuarto y último lugar, se encuentra el diseño de la denominada new competition tool, su ámbito (si está dirigida exclusivamente a servicios digitales o incluye más servicios), el tipo de problemas de competencia que abordará, su forma legal y, una vez más, el modelo institucional previsto. Entre las opciones estudiadas para su implementación se contempla el diseño de un nuevo instrumento legal para la realización de investigaciones de mercado.

La propuesta deberá resolver el potencial solapamiento entre la herramienta ex ante y la nueva herramienta de competencia, si finalmente la Comisión propone la implementación de ambas.

Un tema muy relevante es el diseño institucional, que incluye el reparto de competencias entre el nivel europeo y las autoridades reguladoras y/o de competencia en los Estados miembros. Por lo que se conoce por el momento, el hecho de que las grandes plataformas tengan un ámbito transnacional lleva a que el nivel europeo juegue un papel muy relevante en la aplicación de la regulación que se diseñe, sin perjuicio del papel que puedan jugar las autoridades nacionales en los detalles de aplicación o el tratamiento de determinados casos o ámbitos de aplicación. En el caso español, la
CNMC, al ser autoridad de competencia, de supervisión del sector audiovisual y regulador ex ante de sectores conexos como las telecomunicaciones, está en una situación óptima para el tratamiento integral de los distintos aspectos que tratará el paquete regulatorio que finalmente apruebe el Parlamento Europeo y el Consejo Europeo. Participa, también, en el debate regulatorio tanto directamente como a través de las redes de regulación y competencia (BEREC, ECN y ERGA) de las que es miembro.

Por último, conviene indicar que la consulta pública del Acta de Servicios Digitales incluía aspectos adicionales interesantes como la regulación de smart contracts o el marco laboral de los trabajadores de las plataformas digitales de intermediación.

En lo que respecta al trabajo del Parlamento Europeo, varios comités han adoptado resoluciones sobre la regulación de plataformas digitales que han sido publicados muy recientemente: el comité IMCO sobre mercado interno y protección a los consumidores (Parlamento Europeo, 2020a), el comité JURI de asuntos legales (Parlamento Europeo, 2020b) y el comité LIBE sobre libertades civiles, justicia y asuntos internos (Parlamento Europeo, 2020c). Los textos adoptados convergen en la necesidad de mantener los principios básicos de la directiva de comercio electrónico (los intermediarios online continuarían disfrutando de un régimen de exención de responsabilidad y no estarían obligados a supervisar los contenidos que intermedian), el establecimiento de procedimientos armonizados para eliminar contenidos ilegales, dar más control a los usuarios sobre los datos y reforzar la cooperación entre Estados miembros.

Sobre la regulación económica de plataformas digitales, el comité IMCO es partidario de facilitar que las autoridades de regulación $\triangleright$ 
puedan imponer obligaciones de prohibición de determinados comportamientos como la priorización de servicios propios en la intermediación (self-preferencing) para plataformas con elevado poder de intermediación, o establecer directamente obligaciones como la no discriminación o la transparencia a estas plataformas con elevado poder de negociación. EI informe del comité LIBE trata temas de especial interés y actualidad como el uso de plataformas en campañas electorales y para propaganda política.

En definitiva, la regulación de las plataformas digitales en todos sus aspectos está en pleno proceso de diseño a nivel europeo y en los próximos meses se conocerá la propuesta de la Comisión Europea. La relevancia de estas plataformas y el abanico de aspectos que cubrirá la propuesta hace que sea uno de los temas regulatorios clave que puede configurar la evolución de estas plataformas en la próxima década.

\section{Conclusiones}

Las plataformas digitales tienen una importancia creciente en la articulación de las esferas de comunicación interpersonal y actividad económica de los ciudadanos. Sus extraordinarias capacidades de prestación de servicios innovadores de intermediación han permitido a ciudadanos y a empresas beneficiarse de los avances que plantean en términos de comodidad, abaratamiento de costes y ampliación de oferta. Las características específicas de estas plataformas plantean retos regulatorios importantes en aspectos diversos como la competencia, el acceso a contenidos o la privacidad de los datos.

En los últimos años se ha abierto un amplio debate en el mundo al que no es ajeno la
Unión Europea, cuyas instituciones se encuentran en proceso de diseño del modelo regulatorio de estas plataformas para adaptarse a los retos del siglo xxı. En diciembre se conocerá la propuesta de la Comisión Europea, estando previsto un intenso debate en las instituciones europeas que configurará la arquitectura regulatoria para las plataformas digitales en Europa.

\section{Bibliografía}

Australian Competition and Consumer Commission, ACCC (2019). Digital Platforms Enquiry Final Report. https://www.accc.gov.au/focus-areas/inquiries-ongoing/digital-platforms-inquiry/finalreport-executive-summary

Body of European Regulators for Electronic Comunications, BEREC (2020). BEREC Response to the Public Consultations on the Digital Services Act Package and the New Competition Tool. BoR (20) 138. Septiembre, 2020. https://berec.europa.eu/eng/document_register/subject_matter/ berec/others/9411-berec-response-to-the-publicconsultation-on-the-digital-services-act-package-and-the-new-competition-tool

Bundeskartellamt (2019). Bundeskartellamt prohibits Facebook from combining user data from different sources [press release]. https://www. bundeskartellamt.de/SharedDocs/Meldung/EN/ Pressemitteilungen/2019/07_02_2019_Facebook.html

Bourreau, M., \& De Streel, A. (2019). Digital conglomerates and EU competition policy. SSRN 3350512. https://dx.doi.org/10.2139/ssrn.3350512

Bourreau, M., \& De Streel A. (2020). BigTech acquisitions. Competition \& innovation effects and EU merger control. Centre on Regulation in Europe (CERRE). https://cerre.eu/publications/big-techacquisitions-competition-and-innovation-effectseu-merger-control/ 
Broughton, S., Krämer, J., \& Schnurr, D. (2020). The role of data for digital markets contestability: case studies and data access remedies. Centre on Regulation in Europe (CERRE). https://cerre. eu/publications/data-digital-markets-contestability-case-studies-and-data-access-remedies/

Comisión Europea (2000). Directive 2000/31/EC of the European Parliament and of the Council of 8 June 2000 on certain legal aspects of information society services, in particular electronic commerce, in the Internal Market (Directive on electronic commerce). https://ec.europa.eu/digital-single-market/en/e-commerce-directive

Comisión Europea (2017). Commission fines Google €2.42 billion for abusing dominance as search engine by giving illegal advantage to own comparison shopping service [press release]. https://ec.europa.eu/commission/presscorner/detail/en/IP_17_1784

Comisión Europea (2018). Commission fines Google €4.34 billion for illegal practices regarding Android mobile devices to strengthen dominance of Google's search engine. https://ec.europa. eu/commission/presscorner/detail/en/ IP_18_4581

Comisión Europea (2019a). Antitrust: Commission fines Google $€ 1.49$ billion for abusive practices in online advertising [press release]. https:// ec.europa.eu/commission/presscorner/detail/ en/IP_19_1770

Comisión Europea (2019b). Antitrust: Commission opens investigation into possible anti-competitive conduct of Amazon [press release]. https:// ec.europa.eu/commission/presscorner/detail/ en/IP_19_4291

Comisión Europea (2020b). The Digital Service Act package. https://ec.europa.eu/digital-single-market/ en/digital-services-act-package

Comisión Europea (2020c). Digital Services Act Package. 21st meeting of the eCommerce Expert Group, 26 May 2020. https://ec.europa.eu/ transparency/regexpert/index.cfm?do=groupDetail.groupMeetingDoc\&docid $=41347$
Comisión Europea (2020d). The New Competition Tool. https://ec.europa.eu/info/law/better-regulation/have-your-say/initiatives/12416-Newcompetition-tool

Competition \& Markets Authority, CMA (2020). Online platforms and digital advertising. Market study Final. https://assets.publishing.service. gov.uk/media/5efc57ed3a6f4023d242ed56/Final_report_1_July_2020_.pdf

Condorelli, D., \& Padilla, J. (2020). Harnessing Platform Envelopment in the Digital World. Journal of Competition Law \& Economics, 16(2), 143-187. Abril de 2020. https://doi.org/10.1093/joclec/ nhaa006

Crémer, J., de Montjoye, Y. A., \& Schweitzer, H. (2019). Competition policy for the digital era. Report for the European Commission. https://ec.europa.eu/competition/publications/reports/ kd0419345enn.pdf

De Streel, A., y Feasey, R. (2020). Data Sharing for Digital Markets Contestability: Towards a Governance Framework. CERRE Policy Report. https:// cerre.eu/publications/data-sharing-digital-marketscompetition-governance/

De Streel, A., y Huvosec, M. (2020). The e-commerce Directive as the cornerstone of the internal market. Department for Economic, Scientific and Quality of Life Policies (elaborado a petición del comité IMCO del Parlamento Europeo). https:// www.europarl.europa.eu/RegData/etudes/ STUD/2020/648797/IPOL_STU(2020)648797 _EN.pdf

Department of Justice United States of America. (2020). Justice Department Sues Monopolist Google For Violating Antitrust Laws [press release]. https://www.justice.gov/opa/pr/justicedepartment-sues-monopolist-google-violating-antitrust-laws

European Regulators Group for Audiovisual Media Services, ERGA (2020). ERGA Position Paper on the Digital Services Act. https://erga-online. eu/wp-content/uploads/2020/06/ERGA_SG1_ DSA_Position-Paper_adopted.pdf 
Feasey, R., \& Krämer, J. (2019). Implementing effective remedies for anti-competitive intermediation bias on vertically integrated platforms. Centre on Regulation in Europe (CERRE). https://cerre.net/ publications/implementing-effective-remediesanti-competitive-intermediation-bias-vertically/

Furman, J., Coyle, D., Fletcher, A., McAules, D., \& Marsden, P. (2019). Unlocking digital competition. Report of the digital competition expert panel for the Government of the United Kingdom. https://assets.publishing.service.gov.uk/government/uploads/system/uploads/attachment_data/ file/785547/unlocking_digital_competition_furman_review_web.pdf

Gobierno del Reino Unido (2019). Online Harms White Paper. https://www.gov.uk/government/ consultations/online-harms-white-paper

Krämer, J., Senellart, P., \& De Streel, A. (2020). Making data portability more effective for the digital economy. Centre on Regulation in Europe (CERRE). https://cerre.eu/publications/report-makingdata-portability-more-effective-digital-economy/

Scott Morton, F., Bouvier, P., Ezrachi, A., Jullien, B., Katz, R., Kimmelman, G., Melamed, D., \& Morgenstern, J. (2019). Committee for the Study of Digital Platforms: Market Structure and Antitrust Subcommittee Report. Stigler Center for the Study of the Economy and the State, University of Chicago Booth School of Business. https:// www.judiciary.senate.gov/imo/media/doc/market-structure-report\%20-15-may-2019.pdf
Organización para la Cooperación y el Desarrollo, OCDE (2019). An Introduction to Online Platfor$m s$ and Their Role in the Digital Transformation. OECD Publishing. https://doi.org/10.1787/53e 5f593-en ${ }^{\circ}$

Parlamento Europeo (2020a). Internal Market and Consumer Protection (IMCO) committee. Digital Services Act - Improving the functioning of the Single Market. Septiembre de 2020. https://www. europarl.europa.eu/doceo/document/TA-9,2020-0272_EN.html

Parlamento Europeo (2020b). Legal Affairs (JURI) committee. Report on the Digital Services Act: adapting commercial and civil law rules for commercial entities operating online. Octubre de 2020. https://www.europarl.europa.eu/doceo/document/TA-9-2020-0273_EN.html

Parlamento Europeo (2020c). Civil Liberties, Justice and Home Affairs (LIBE) committee. Report on the Digital Services Act and fundamental rights issues posed. Octubre de 2020. https://www.europarl.europa.eu/doceo/document/TA-9-20200274_EN.html

Whish, R. (2020e). New Competition Tool: Legal comparative study of existing competition tools aimed at addressing structural competition problems with a particular focus on the UK's market investigation tool. Comisión Europea. https:// ec.europa.eu/competition/consultations/2020_ new_comp_tool $/ \mathrm{kd} 0420573 \mathrm{enn}$. pdf 